\title{
Anisotropies of tactile distance perception on the face
}

\author{
Matthew R. Longo ${ }^{1}$ • Elena Amoruso ${ }^{1}$ - Elena Calzolari ${ }^{2}$ - Michael Ben Yehuda ${ }^{3}$ - Patrick Haggard ${ }^{4}$ • Elena Azañón ${ }^{5,6,7}$
}

Published online: 2 July 2020

(C) The Psychonomic Society, Inc. 2020

\begin{abstract}
The distances between pairs of tactile stimuli oriented across the width of the hand dorsum are perceived as about $40 \%$ larger than equivalent distances oriented along the hand length. Clear anisotropies of varying magnitudes have been found on different sites on the limbs and less consistently on other parts of the body, with anisotropies on the center of the forehead, but not on the belly. Reported anisotropies on the center of the forehead, however, might reflect an artefact of categorical perception from the face midline, which might be comparable to the expansion of tactile distance perception observed for stimuli presented across joint boundaries. To test whether tactile anisotropy is indeed a general characteristic of the tactile representation of the face, we assessed the perceived distance between pairs of touches on the cheeks and three locations on the forehead: left, right, and center. Consistent with previous results, a clear anisotropy was apparent on the center of the forehead. Importantly, similar anisotropies were also evident on the left and right sides of the forehead and both cheeks. These results provide evidence that anisotropy of perceived tactile distance is not a specific feature of tactile organization at the limbs but it also exists for the face, and further suggest that the spatial distortions found for tactile distances that extend across multiple body parts are not present for stimuli that extend across the body midline.
\end{abstract}

Keywords Distance perception $\cdot$ Anisotropy $\cdot$ Categorical perception $\cdot$ Face $\cdot$ Tactile perception

\section{Introduction}

In one of the first systematic investigations of the sense of touch in the nineteenth century, Weber (1834) found that as he moved the two points of a compass across his skin it felt to him as if the distance between them increased as he moved

Elena Azañón

eazanyon@gmail.com

1 Department of Psychological Sciences, Birkbeck, University of London, London, UK

2 Neuro-Otology Unit, Division of Brain Sciences, Imperial College London, London, UK

3 Department of Experimental Psychology, University of Oxford, Oxford, UK

4 Institute of Cognitive Neuroscience, University College London, London, UK

5 Institute of Psychology, Otto-von-Guericke University Magdeburg, Magdeburg, Germany

6 Center for Behavioral Brain Sciences, Otto-von-Guericke University Magdeburg, Magdeburg, Germany

7 Department of Behavioral Neurology, Leibniz Institute for Neurobiology, Magdeburg, Magdeburg, Germany them from a region of relatively low sensitivity (e.g., the forearm) to a region of relatively higher sensitivity (e.g., the hand). This pattern has been replicated in numerous subsequent studies (Anema, Wolswijk, Ruis, \& Dijkerman, 2008; Cholewiak, 1999; Goudge, 1918; Miller, Longo, \& Saygin, 2016; TaylorClarke, Jacobsen, \& Haggard, 2004), which collectively demonstrate a systematic relation between tactile sensitivity and perceived tactile distance on the skin. Analogous illusions can also be shown for stimuli in different orientations on a single skin surface (e.g., Fiori \& Longo, 2018; Green, 1982; Longo \& Haggard, 2011). For example, Longo and Haggard (2011) found that tactile distances oriented across the width of the hand dorsum are perceived as about $40 \%$ larger than equivalent distances oriented along hand length.

Such anisotropies in perceived tactile distance have been reported on several skin surfaces. In addition to the hand dorsum (Canzoneri et al., 2013; Longo \& Golubova, 2017; Longo \& Haggard, 2011; Longo \& Sadibolova, 2013; Miller, Longo, \& Saygin, 2014), other studies have reported anisotropies on the forearm (Green, 1982; Le Cornu Knight, Longo, \& Bremner, 2014), the thigh (Green, 1982), and the shin (Stone, Keizer, \& Dijkerman, 2018). Intriguingly, in each of these cases, the direction of the anisotropy is similar, with distances perceived as larger when oriented across the width 
of the limbs than when oriented along their length. Longo and Haggard (2011) suggested that both the classic form of Weber's illusion and such anisotropies could result from the geometry of tactile receptive fields in the somatosensory cortex, which in addition to being smaller on sensitive skin surfaces (Mountcastle, 2005; Sur, Merzenich, \& Kaas, 1980), are generally oval-shaped on the limbs with the long axis of the receptive field aligned with the long axis of the limb (Alloway, Rosenthal, \& Burton, 1989; Brooks, Rudomin, \& Slayman, 1961). Interestingly, anisotropies on the glabrous skin of the palm are substantially smaller or even absent (Fiori \& Longo, 2018; Le Cornu Knight et al., 2014; Longo, Ghosh, \& Yahya, 2015), which is consistent with the idea that tactile receptive fields on the palm are circular, and when ovalshaped without a consistent orientation (DiCarlo \& Johnson, 2002; DiCarlo, Johnson, \& Hsiao, 1998). Moreover, anisotropies of tactile distance differ in magnitude across body parts, which have been found for example to be larger on the forearm than on the hand dorsum (Le Cornu Knight et al., 2014). Thus, assessing the anisotropy of tactile distance across body parts is a powerful tool for assessing the structure of tactile spatial perception across the body.

Interestingly, there does not appear to be anisotropy of tactile distance on the belly (Green, 1982; Longo, Lulciuc, \& Sotakova, 2019; Marks et al., 1982), consistent with the interpretation that tactile distance anisotropy is a specific characteristic of the limbs, with their highly elongated shape. Two recent studies, however, have reported anisotropies of tactile distance perception on the face (Fiori \& Longo, 2018; Longo et al., 2015). Longo et al. (2015) used a two-alternative forcedchoice paradigm and found that tactile distances oriented across the width of the forehead (i.e., the ear-to-ear axis) were perceived as about $20 \%$ larger than comparable distances oriented with the height of the forehead (i.e., the chin-to-forehead axis). Fiori and Longo (2018) asked participants to make verbal size estimates of single tactile distances, and also found an anisotropy with distance across the forehead perceived as larger. However, one notable aspect of both these studies is that they presented stimuli at the center of the forehead. This means that stimuli presented across the width of the forehead, but not along its height, included one touch on each side of the body midline. It is therefore possible that tactile distance anisotropies on the forehead might indeed reflect an artifact of the across stimuli straddling the face midline, rather than a general characteristic of the tactile representation of the face. Several studies have found perceptual expansion of tactile distances that cross joint boundaries (de Vignemont, Majid, Jola, \& Haggard, 2008; Le Cornu Knight, Cowie, \& Bremner, 2017; Le Cornu Knight et al., 2014). These studies have found that pairs of tactile stimuli straddling the wrist are overestimated in distance beyond what would be expected given judgments on the adjacent regions of the forearm and hand, indicating a form of categorical perception based on segmentation of the body into discrete parts. In this regard, the body midline could - like joints - produce categorical perception effects for stimuli falling on opposite sides, given the bilateral symmetry of the human body and the fact the tactile signals from each side of the body are sent primarily to the contralateral cerebral hemisphere (Mountcastle, 1957; Penfield \& Boldrey, 1937).

To our knowledge no previous studies have investigated a potential effect of the body midline on tactile distance perception. Note that the lack of anisotropy at the center of the belly in previous studies (Green, 1982; Longo et al., 2019; Marks et al., 1982) is not indicative per se of the lack of midline categorical effects, as these studies did not test anisotropy at the side of the belly for comparison. Hence, the reported lack of anisotropy at the center of the belly could be the net combination of positive categorical perception plus a reverse anisotropy effect, where the across stimuli are actually perceived as being shorter than the along stimuli.

In this study, we investigated whether there are anisotropies of perceived tactile distance on the face independent of potential categorical perception induced by the body midline to (1) clarify whether body midline can affect perception in the same way as joint boundaries can, and (2) provide a detailed description of distortions of tactile space in five regions of the face. In Experiment 1, we compared perceived tactile distances across versus along the left and right sides of the forehead (i.e., entirely on one side of the midline) and the left and right cheeks. In Experiment 2, we directly compared stimuli presented centered on the forehead to stimuli presented entirely on the left or right sides.

\section{Experiment 1}

In the first experiment, we measured anisotropies on the left and right sides of the forehead and cheeks. Because all stimuli were presented entirely on one side of the body midline, any potential effect of categorical perception based on the midline should not affect results.

\section{Methods}

Participants Twenty-two people (11 women, mean age: 24.0 years, range: 18-35) participated for payment after giving written informed consent. Procedures were approved by the local ethics committee. Testing started on an additional participant but ended after ten trials due to a technical problem and this participant was excluded. All the participants took part in a second experiment (not involving touch), either on the same day (following the anisotropy task) or a week apart. The data from this experiment are not considered here. Data from three participants were excluded due to poor fitting of the data in one or more conditions (see below). 
The two previous studies that investigated tactile distance anisotropies on the face found effect sizes of Cohen's $d$ of 1.11 (Longo et al., 2015) and 1.35 (Fiori \& Longo, 2018), respectively. Averaging these numbers weighted by their sample sizes (35 and 25) gives an average effect size of Cohen's $d$ $=1.21$. A power analysis using G*Power 3.1 software (Faul, Erdfelder, Land, \& Buchner, 2007) found that eight participants were required for a power of 0.80 and an alpha of 0.05 . Our sample size was therefore well powered to identify comparable effects.

Procedure The stimuli were pairs of wooden sticks that tapered to a point $(\sim 1 \mathrm{~mm})$ that were embedded in foamboard at distances of 2, 2.5, 3, or $4 \mathrm{~cm}$ apart, similar to those we have used in other studies (e.g., Calzolari, Azañón, Danvers, Vallar, \& Longo, 2017; Fiori \& Longo, 2018; Hidaka, Tucciarelli, Azañón, \& Longo, 2020; Longo \& Haggard, 2011). On each trial, two pairs of stimuli were applied to the participant's face, one with the two touches oriented across the width of the face (i.e., the ear-to-ear axis) and one oriented along the length of the face (i.e., the chin-to-forehead axis). Each stimulus was applied manually by an experimenter for approximately $1 \mathrm{~s}$ with an inter-stimulus interval of approximately $1 \mathrm{~s}$. The participant's task was to judge whether the distance between the two points felt farther apart in the along or the across orientation by making an unspeeded verbal judgment. Across trials, there were seven pairs of distances used, varying in the ratio of distances in the two orientations (across/along): $2 / 4 \mathrm{~cm}, 2 / 3$ $\mathrm{cm}, 2.5 / 3 \mathrm{~cm}, 3 / 3 \mathrm{~cm}, 3 / 2.5 \mathrm{~cm}, 3 / 2 \mathrm{~cm}$, and $4 / 2 \mathrm{~cm}$.

In different blocks, stimuli were applied to four different locations on the face: the left and right sides of the forehead and the left and right cheeks. On the forehead, we identified the location on each side of the forehead midway between the facial midline (i.e., an upwards continuation of the midline of the nose) and the temple, which formed the center point for stimuli. For the cheeks, stimuli were applied roughly midway between the lateral edge of the nose and the ear tragus.

There were four experimental blocks, one for each location on the face, which were presented in random order for each participant. Each block consisted of 56 trials, eight trials for each of the seven ratios between the across and along distances, half with the across stimulus presented first and half with the along stimulus presented first. The 56 trials in each block were presented in random order. If a given stimulus was perceived as a single touch or as a pair of non-simultaneous touches, that trial was cancelled and repeated at the end of the condition. This procedure was added in case a given participant had a two-point discrimination threshold larger than the smaller of our stimuli (i.e., $2 \mathrm{~cm}$ ) in a given orientation or location on the face. However, participants overall reported the perception of one (or non-simultaneous stimulation) in less than $1 \%$ of the trials $(\mathrm{M}=0.79 \%)$, which were removed from analyses and re-tested at the end. Regardless of this, the mean two point-point discrimination threshold reported in several studies at the cheeks and forehead is well below $2 \mathrm{~cm}$ (Mancini et al., 2014; Sato, Okada, Miyamoto, \& Fujiyama, 1999; Vriens \& van der Glas, 2009; Won, Kim, Kim, \& Kim, 2017). Nevertheless, it does remain possible that on some trials participants may have felt only one touch for one of the stimuli, which could affect the nature of the judgment they made. Participants were allowed to take a short break between blocks. They were blindfolded during the experiment.

Analysis We analyzed the proportion of trials in which the tactile distance across the width of the face was judged as larger as a function of the ratio of the across and along stimuli, plotted logarithmically to produce a symmetric distribution around a ratio of 1 (i.e., the ratio at which the two distances were the same size). Data from each skin region in each participant was fit with a cumulative Gaussian function using maximum-likelihood estimation with the Palamedes toolbox (Prins \& Kingdom, 2009) for MATLAB (Mathworks, Natick, MA, USA).

The criteria for exclusion of participants was if the $\mathrm{R}^{2}$ for the psychometric functions was less than 0.5 in any of the four skin regions, as in other recent studies from our lab (Longo, 2017; Longo et al., 2015; Longo \& Morcom, 2016). Three participants were excluded on this basis.

The cumulative Gaussian curve fit to the data has two parameters, the mean and the standard deviation. The mean of the function indicates the point of subjective-equality (PSE), which is the ratio between the across and along stimuli for which participants were equally likely to say that each was bigger. If there is no anisotropy, then the PSE should on average be equal to 1 (i.e., the stimuli should feel the same when they really are the same). If there is a perceptual bias for stimuli to be perceived as bigger when oriented with face height than with face width, then PSEs should on average be larger than 1 (i.e., the stimuli would be perceived as the same size when the across stimulus was bigger); in contrast, if there is a bias for stimuli to be perceived as bigger when oriented with face width than face height, then PSEs should be less than 1 (i.e., the stimuli would be perceived as the same size when the along stimulus was bigger). The second parameter of the psychometric function, the standard deviation, is inversely related to the slope of the psychometric function, and therefore to the precision of responses.

To assess anisotropy, we conducted one-sample t-tests comparing mean PSE to a ratio of 1 . Note that all statistical tests were performed on the logarithms of PSEs, which were converted back to ratios for reporting mean values. To compare the different skin surfaces, we conducted a $2 \times 2$ factorial analysis of variance (ANOVA) including region (forehead, cheek) and laterality (left, right) as withinsubject factors and both PSEs and standard deviations as dependent variables. 
As measures of effect size, we calculated Cohen's $d$ for one-sample t-tests, $d_{z}$ for paired t-tests, and $\eta_{\mathrm{p}}{ }^{2}$ for F-statistics.

\section{Results and discussion}

The results from Experiment 1 are shown in Fig. 1. $R^{2}$ values indicated a good fit to the data, with psychometric functions accounting for $86.2 \%$ of the variance on the forehead and $92.3 \%$ on the cheek. To investigate the presence of anisotropy on the forehead and cheek, we first compared mean PSEs to 1 collapsing across the left and right sides. There was a clear anisotropy on the forehead (M: 0.884), $t(18)=-4.71, p<$ $0.0005, d=1.081$, with tactile distances oriented across the width of the forehead perceived as larger than those oriented along forehead height. There was also a clear anisotropy in the same direction on the cheek (M: 0.889), $t(18)=-3.99, p<$ $0.001, d=0.915$.

An ANOVA on PSEs revealed a modest effect of laterality, $F(1,18)=4.63, p=0.045, \eta_{\mathrm{p}}{ }^{2}=0.205$, with larger anisotropy on the left ( $M: 0.860)$ than on the right side of the face $(M$; $0.913)$. There was no main effect of skin region, $F(1,18)=$ $0.02, p>0.50, \eta_{\mathrm{p}}{ }^{2}=0.001$, nor an interaction of region and laterality, $F(1,18)=0.33, p>0.50, \eta_{\mathrm{p}}{ }^{2}=0.018$.

An ANOVA on the standard deviations of the psychometric functions revealed a significant main effect of body part, $F(1,18)=16.94, p<0.001, \eta_{\mathrm{p}}{ }^{2}=0.485$, with smaller standard deviations (i.e., higher sensitivity) on the cheek than the forehead. There was also a significant main effect of laterality,
$F(1,18)=8.35, p=0.010, \eta_{\mathrm{p}}{ }^{2}=0.317$, with smaller standard deviations on the right side of the face than on the left side. There was no significant interaction between body part and laterality, $F(1,18)=1.88, p=0.187, \eta_{\mathrm{p}}{ }^{2}=0.095$.

This experiment replicates the anisotropies on the forehead reported in previous studies (Fiori \& Longo, 2018; Longo et al., 2015). Critically, because in contrast to those previous studies, stimuli were presented entirely on one side of the face midline, these results demonstrate further that this effect does not require that the across stimuli straddle the face midline.

\section{Experiment 2}

The results from Experiment 1 demonstrate that tactile distance anisotropy on the forehead exists independent of any potential effect of categorical perception from the face midline. However, the magnitude of anisotropy found on the sides of the forehead in Experiment 1 ( $M: 0.884)$ is somewhat smaller in magnitude than that found previously on the center of the forehead (M: 0.818; Longo et al., 2015). This is consistent with the possibility that there may be a categorical perception effect from the face midline that modulates the magnitude of anisotropy. To investigate this possibility, Experiment 2 compared anisotropy at the center of the forehead and on the right and left sides of the forehead in the same participants. a

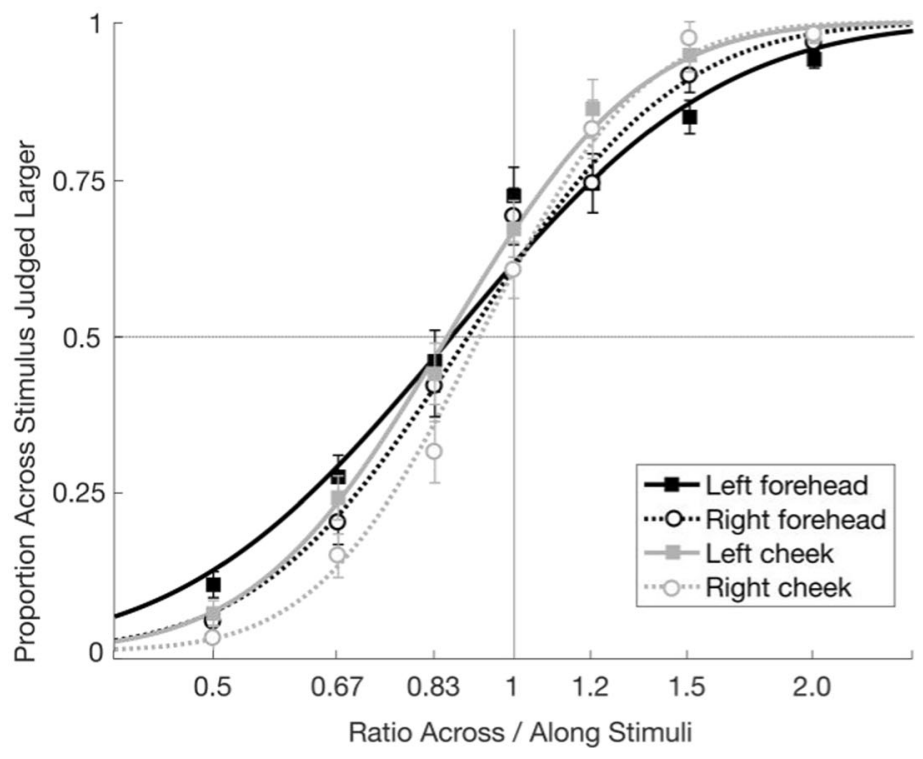

b

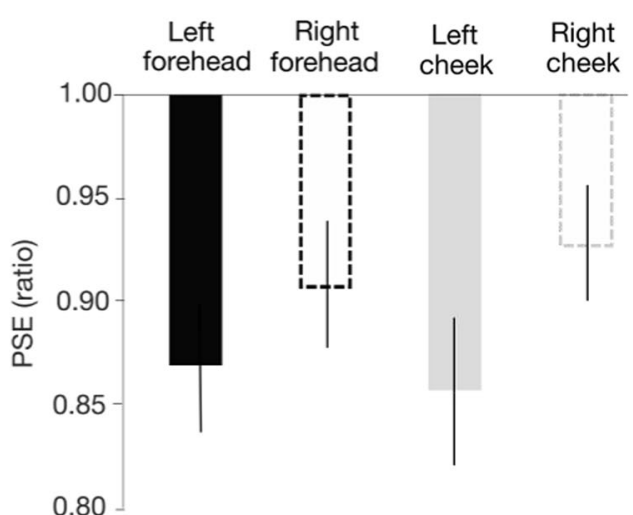

Fig. 1 Results from Experiment 1. (a) Proportion of "across" stimuli judged as being larger as a function of the presented ratio (Across/ Along). Curves are cumulative Gaussian function fits of the data. All four locations showed anisotropy, with tactile distances oriented across the width of the face perceived as larger than those oriented along the height of the face. (b) Ratio between the across and along stimuli for which participants were equally likely to say that each was bigger at each condition (i.e., point of subjective equality, PSE; all PSEs $<1$; all $p<$ 0.025). Error bars represent the standard error of the mean 


\section{Participants}

Thirty people (18 women, mean age: 27.6 years, range: 20 $45)$ participated after giving written informed consent. To our knowledge there are five previous experiments that have measured categorical perception effects on tactile distance (all at the wrist): Experiments 1 and 2 of de Vignemont et al. (2008), Experiments 1 and 2 of Le Cornu Knight et al. (2014), and the single experiment reported by Le Cornu Knight et al. (2017). For each of these experiments, we calculated the effect size (Cohen's $d_{z}$ ) for the key comparison of stimuli crossing the wrist to stimuli on the hand. We calculated an average of these effect sizes weighted by the sample size of each experiment, which yielded a mean effect size of Cohen's $d_{z}=0.590$. A power analysis using $\mathrm{G}^{*}$ Power 3.1 for a two-tailed t-test with this effect size, power of 0.80 , alpha of 0.05 , indicated that 25 participants were required. Our sample is therefore appropriately powered to identify a comparable effect of the body midline.

\section{Methods}

Stimuli were similar to those used in Experiment 1. Across trials, stimuli were presented at three locations on the forehead. The left and right forehead locations were defined as in Experiment 1, while the center location was in the middle of the forehead, straddling the facial midline, consistent with the location of stimuli in the two other studies that investigated tactile distance on the face (Fiori \& Longo, 2018; Longo et al., 2015). There were five pairs of distances used (across/along): $2 / 4 \mathrm{~cm}, 2 / 3 \mathrm{~cm}, 3 / 3 \mathrm{~cm}, 3 / 2 \mathrm{~cm}$, and $4 / 2 \mathrm{~cm}$, as in previous studies from our lab (e.g., Calzolari et al., 2017; Longo et al., 2015; Longo \& Haggard, 2011). The participant's task was to judge whether the distance between the two touches felt bigger for the first or for the second stimulus, rather than indicating whether the along or the across stimulus was larger (as in Experiment 1). This change reduces the likelihood that any biases observed in Experiment 1 might have been due to response bias, to preferentially respond "across," rather than perceptual bias.

There were seven blocks of 30 trials each. Each block included two repetitions of each pair of distances (14 in total), one with the across stimulus first another with the along stimulus first, at each of the three forehead locations. The order of trials was randomized in each block. Again, a trial was repeated (at the end of the block) if the participant reported feeling one touch rather than two or two asynchronous touches $(0.38 \%)$.

\section{Analysis}

The analysis was similar to that of Experiment 1. We applied the same exclusion criteria (i.e., $R^{2}<0.5$ in any condition), but no participant was excluded. One-sample t-tests were used to compare mean PSE in each condition to a ratio of 1. A oneway repeated-measures analysis of variance (ANOVA) was used to compare the three conditions. Mauchley's test revealed no violation of the sphericity assumption.

\section{Results and discussion}

The results from Experiment 2 are shown in Fig. 2. Overall, the psychometric functions showed good fit to the data, with mean $R^{2}$ indicating that they accounted for $95.4 \%, 94.7 \%$, and $96.5 \%$ of the variance in the center, left, and right locations, respectively.

A significant anisotropy was apparent at the center of the forehead (M: 0.919), $t(29)=-2.38, p<0.05, d=0.435$. Critically, similar anisotropies were also found on the left side of the forehead (M: 0.857), $t(29)=-4.41, p<0.0005, d=$ 0.806 , and the right side ( $M: 0.912), t(29)=-2.39, p<0.05$, $d=0.437$. An ANOVA revealed no significant differences between the three locations, $F(2,58)=2.50, p=0.09, \eta_{\mathrm{p}}{ }^{2}=$ 0.079 . It is worth noting that of the three testing locations, the numerical magnitude of anisotropy was actually smallest on the center of the forehead, a trend if anything opposite to what would be expected if there were an effect of categorical perception.

Given the laterality effect observed in Experiment 1, we also compared the magnitude of anisotropy on the left and right forehead. This difference was not statistically significant, $t(29)=-1.64, p=0.111, d_{z}=0.300$, but the trend was in the same direction as observed in Experiment 1.

An ANOVA on the standard deviation of the psychometric function revealed a significant effect of stimulus location, $F(2$, 58) $=5.89, p<0.01, \eta_{\mathrm{p}}{ }^{2}=0.169$. Standard deviations were significantly smaller at the center of the forehead than on the left, $t(29)=-2.37, p<0.05, d_{z}=0.432$, or the right, $t(29)=-$ $3.07, p<0.005, d_{z}=0.560$, side. Thus, while there was no evidence that perceptual distances are expanded for stimuli straddling the face midline, sensitivity does appear to be higher near the midline. Given the laterality effect on standard deviations found in Experiment 1, we also compared the left and right sides directly, which did not differ significantly, $t(29)=1.20, p>0.20, d_{z}=0.218$, with the trend going in the opposite direction to that seen in Experiment 1.

The results of this experiment provide further evidence for the existence of tactile distance anisotropies on the forehead. There was no evidence, however, for a categorical perception effect on tactile distance for stimuli crossing the facial midline. This is in interesting contrast to studies that have reported such effects for stimuli crossing joint boundaries (de Vignemont et al., 2008; Le Cornu Knight et al., 2017, 2014). 
a

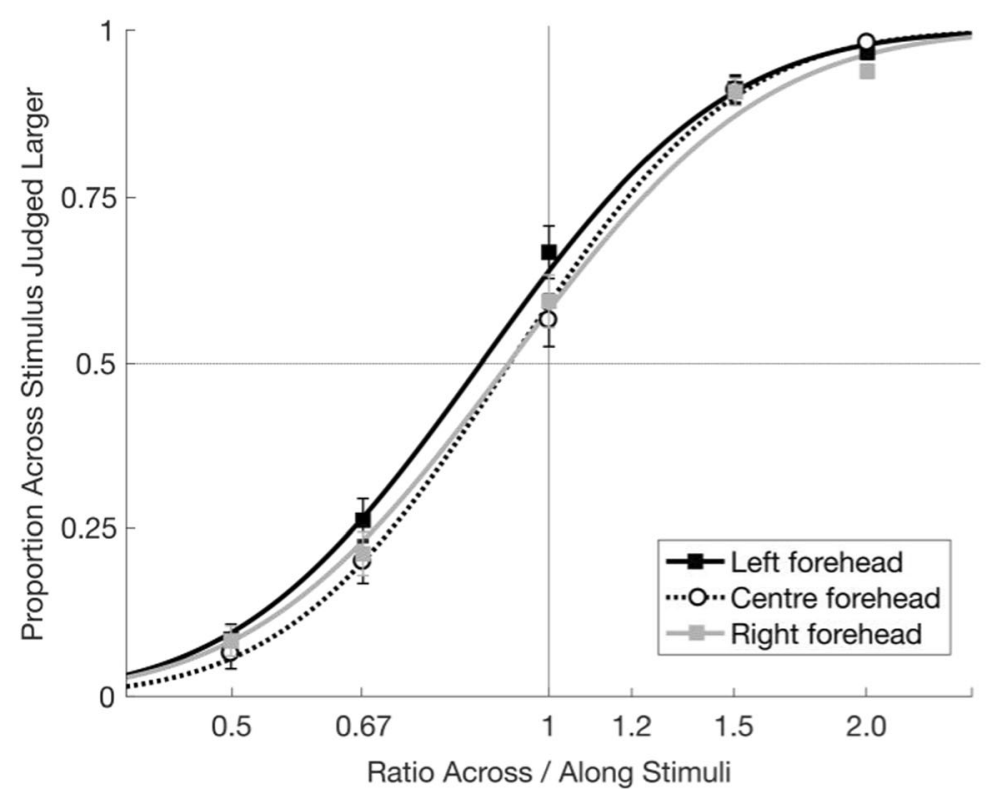

b

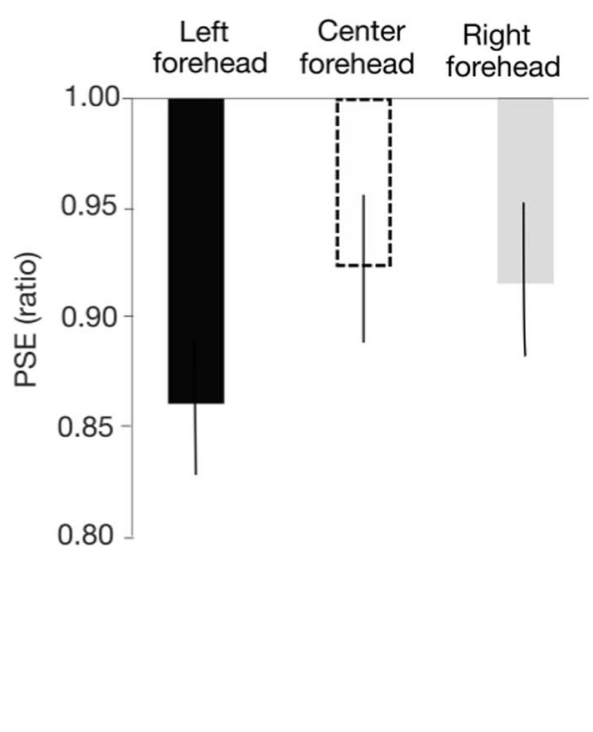

Fig. 2 Results from Experiment 2. (a) Proportion of "across" stimuli judged as being larger as a function of the presented ratio (Across/ Along). Curves are cumulative Gaussian function fits of the data. All three locations on the forehead showed anisotropy. (b) Ratio between

\section{Meta-analysis of studies investigating anisotropy on the forehead}

Four experiments, to our knowledge, have now investigated tactile distance anisotropy on the forehead, the two experiments reported here and two previous studies (Fiori \& Longo, 2018; Longo et al., 2015). To aggregate information across these studies, we therefore conducted a random-effects meta-analysis using the metafor package (Viechtbauer, 2010) for R 3.4.3 software. The study of Longo et al. (2015) used a two-alternative forced-choice method, with anisotropy quantified as the PSE of the psychometric function, as in the two studies reported here. In contrast, in the study of Fiori and Longo (2018), participants made verbal estimates of the size of stimuli in different orientations, with anisotropy quantified as the ratio of judged size in the across and along orientations. Because of this difference in methods, we conducted the metaanalysis on standardized means. Because Cohen's $d$ produces a slight upwards estimation bias, corrected effect sizes (Hedges's g) were used (Borenstein, Hedges, Higgins, \& Rothstein, 2009). In each case, positive values of Hedges's $g$ indicate a bias to judge stimuli as larger in the across orientation, and negative values a bias to judge stimuli as larger in the along orientation. The different stimulus locations in the two experiments reported here were collapsed for this analysis.

Figure 3 shows a forest-plot of the results from the metaanalysis. Overall, there was clear evidence for anisotropy on the forehead, with an overall meta-analytic estimate of a large the across and along stimuli for which participants were equally likely to say that each was bigger at each condition (i.e., point of subjective equality, PSE; all PSEs $<1, p<0.05)$. Error bars represent the standard error of the mean

effect size $(M: 0.987), z=6.58, p<0.0001,95 \% \mathrm{CI}=[0.693-$ 1.281]. There was no evidence for heterogeneity, $Q(3)=4.58$, $p>0.20, I^{2}=37.0 \%$, indicating that the variability between experiments was not larger than would be expected by chance.

\section{General discussion}

The present results provide clear evidence for anisotropies of tactile distance perception on the face, both on the forehead and on the cheek. These results converge with other recent studies (Fiori \& Longo, 2018; Longo et al., 2015) in showing that tactile distances oriented across the width of the face (i.e., the ear-to-ear axis) are perceived as larger than distances oriented along the length of the face (i.e., the chin-to-forehead axis). The present results further demonstrate that such effects on the face are not an artefact of the across stimuli straddling the face midline, as clear anisotropies were found for stimuli on both the forehead and cheeks that were entirely on one side of the midline.

These results add to a growing literature showing tactile distance anisotropies across a wide range of skin regions. In addition to the forehead and cheek, biases to judge tactile distance as larger when aligned with body width than with body length have also been found on the hand dorsum (Longo \& Golubova, 2017; Longo \& Haggard, 2011), the forearm (Green, 1982; Le Cornu Knight et al., 2014), the thigh (Green, 1982), and the shin (Stone et al., 2018). Similar effects 


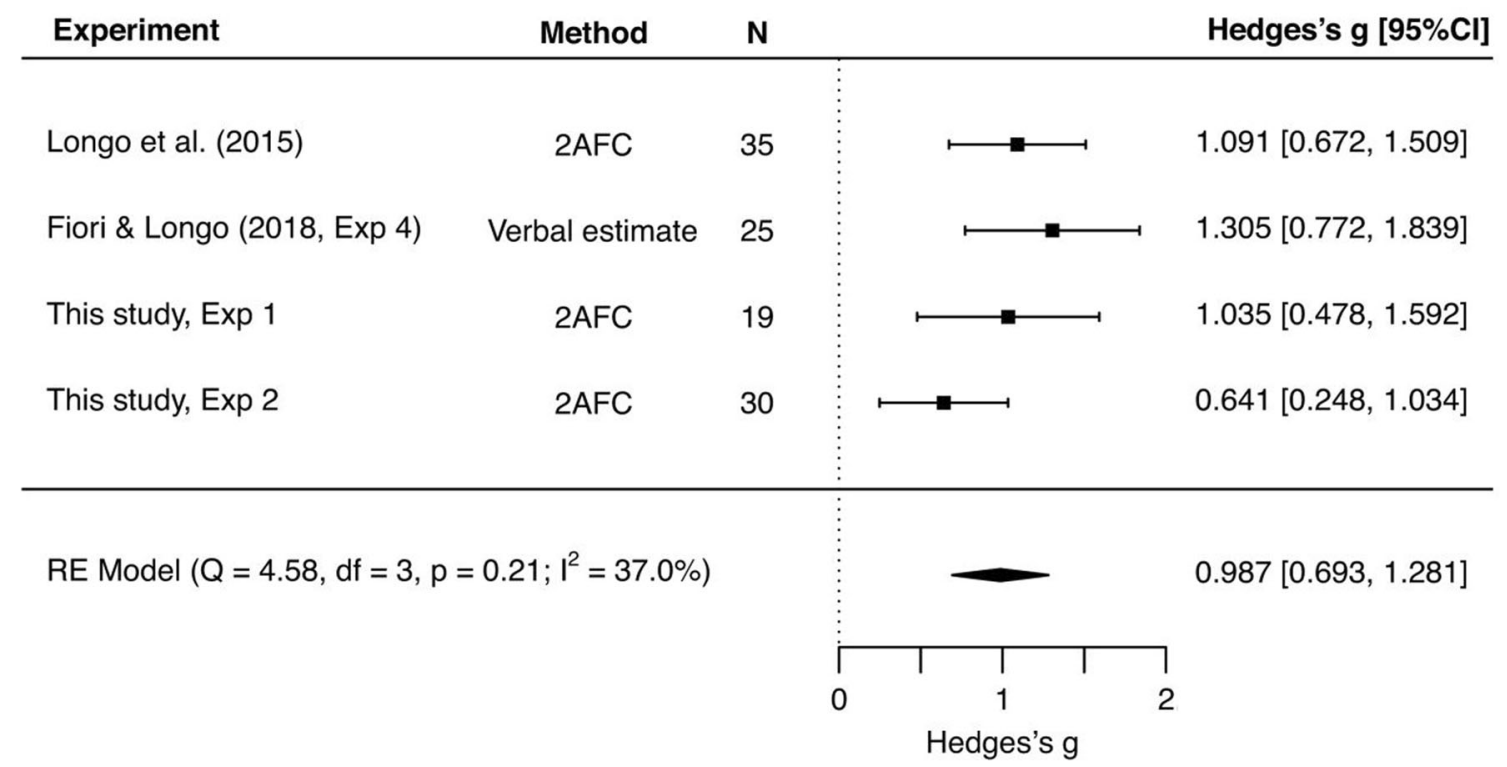

Fig. 3 Forest plot showing results from a random-effects meta-analysis of experiments investigating tactile distance anisotropy on the forehead. Positive values of Hedges's $g$ indicate a bias to judge tactile distances

have also been found on the palm in a few studies (Fiori \& Longo, 2018; Le Cornu Knight et al., 2014; Longo et al., 2015), whereas other studies have found no anisotropy (Green, 1982; Longo \& Golubova, 2017; Longo \& Haggard, 2011). Even when an anisotropy has been found on the palm, however, it has always been substantially smaller than on the dorsum. The one body part on which no anisotropy has been consistently found in healthy participants is the belly (Green, 1982; Longo et al., 2019; Marks et al., 1982), although recent studies suggest that anisotropy on the belly may occur in conditions such as anorexia nervosa (Engel \& Keizer, 2017; Keizer et al., 2011; Keizer, Smeets, Dijkerman, van Elburg, \& Postma, 2012; Spitoni et al., 2015) and obesity (Mölbert et al., 2016; Scarpina, Castelnuovo, \& Molinari, 2014). The presence of anisotropy on the face is therefore notable in that it is the one non-limb body part on which anisotropy has been consistently found. This demonstrates that anisotropy is not a specific characteristic of the limbs, with their highly elongated shape.

Several recent studies have found categorical perception effects of joint boundaries on tactile distance, with tactile distances straddling the wrist boundary overestimated compared to stimuli on the adjacent regions of the forearm and hand (de Vignemont et al., 2008; Le Cornu Knight et al., 2017, 2014). In the current study, not only was the anisotropy in tactile distance perception on the forehead found not to be an artefact of categorical perception from the face midline, we found no evidence for such categorical perception effects at all. This suggests that whereas joint boundaries may induce discontinuities into tactile perceptual experience, the body midline may not. as larger when oriented across the width of the forehead. Collectively, these results provide strong evidence for anisotropy on the forehead

It is important to note that in the case of joints, continuous motion provides repeated sensory feedback about the categorical distinction between body parts, which could explain the altered and biased perception across joints, while this is not the case for the two adjacent skin regions separated through the midline. On the other hand, the lack of categorical perception across the midline may be related to inter-hemispheric communication between somatosensory regions and to the distribution of ipsilateral projections of tactile afferent signals. Neurophysiological studies have found that both ipsilateral responses (Conti, Fabri, \& Manzoni, 1986; Dreyer, Loe, Metz, \& Whitsel, 1975; Iwamura, 2000; Jones \& Powell, 1969a) and callosal projections (Jones \& Powell, 1969b; Killackey, Gould, Cusick, Pons, \& Kaas, 1983; Shanks, Pearson, \& Powell, 1985) are stronger for regions close to the midline on both the torso and the face. Analogous results have been reported in humans using both functional magnetic resonance imaging (fMRI; Fabri, Polonara, Salvolini, \& Manzoni, 2005) and psychophysical (Tamè \& Longo, 2015) methods. This pattern has been traditionally interpreted as a mechanism for binding the representations of the two hemibodies (Jones \& Powell, 1969b; Pandya \& Vignolo, 1969), a process of "midline fusion" (Manzoni, Barbaresi, Conti, \& Fabri, 1989) analogous to that seen in the visual system to merge the two visual hemi-fields (Hubel \& Wiesel, 1967). The absence of categorical perception effects related to the midline may therefore be a result of a specific aspect of somatosensory organization designed to avoid perceptual discontinuities at the midline.

In contrast to the lack of effects of stimulus location on the forehead on anisotropy, there was an effect on the standard 
deviation of the psychometric functions. Sensitivity of discriminating tactile distances was higher for stimuli presented at the center of the forehead than on either the left or the right side. Thus, the facial midline may be associated with higher tactile precision, without inducing any spatial distortions. This may be related to the finding that tactile acuity and the precision of tactile localization are higher in the vicinity of joints than in the center of limbs (e.g., Boring, 1942; Cody, Garside, Lloyd, \& Poliakoff, 2008; Weber, 1834). It is also possible that this enhanced sensitivity near the midline might result directly from the mechanisms described in the previous paragraph. Stimuli near the midline may be processed by mechanisms in both the left and the right somatosensory cortices, whereas more lateral stimuli may be processed more exclusively contralaterally.

The finding of anisotropy on both the cheek and forehead, innervated respectively by the maxillary and ophthalmic branches of the trigeminal nerve, is notable in light of evidence that the representation of the upper and lower regions of the face may be represented differently. Woolsey and colleagues (Ullrich \& Woolsey, 1954; Woolsey, Marshall, \& Bard, 1942) reported that trigeminal inputs in monkeys are represented in two distinct regions of the somatosensory cortex, a main trigeminal region and an "upper head area." Detailed somatotopic maps of the face have found clear segregation of neurons responsive to each of the three divisions of the trigeminal nerve (Dreyer et al., 1975). Similar separation has also been found in the map of the face in New World monkeys (Jain, Qi, Catania, \& Kaas, 2001), with separate areas representing the upper and lower face. In humans, studies using fMRI have found inconsistent patterns of activations with regard to the representations of the face in the primary somatosensory cortex (SI). For instance, Iannetti et al. (2003) found a large overlap, within both SI and the secondary somatosensory cortex (SII), of the foci activated by mechanical stimulation of the forehead (ophthalmic trigeminal division) and lower lip (mandibular trigeminal division). Moulton et al. (2009), on the other hand, found that facial areas stimulated with a brush within an onion-skin layer (i.e., segmenting the face through concentric oval shapes from rostral to caudal) were closely represented in the cortex, even if facial areas were separate and innervated by different branches of the trigeminal nerve. For example, a section of the forehead and cheek, falling putatively into the same onion-skin layer were closely represented in the cortex (see also Dasilva et al., 2002). On the motor side, there are clear double dissociations for apraxia for the lower and upper face (Bizzozero et al., 2000). Perceptual studies of self-face representation based on the relative localization of different face parts have identified independent representations of the upper and lower face (Fuentes, Runa, Blanco, Orvalho, \& Haggard, 2013), which show different patterns of distortion (Mora, Cowie, Banissy, \& Cocchini, 2018). In the present study, however, we found no difference in the nature or magnitude of tactile distance anisotropy on the lower and upper face.

Of course, the anisotropies observed on the cheek and forehead are also similar to those reported on the hand in a number of studies. The qualitatively similar anisotropies found on the face and the hand are intriguing in light of the potential similarities across the shape of receptive fields, as well as functional connections between the representations of these body parts. With regard to the shape of facial receptive fields, there have been several studies focusing on the somatosensory representation of the head and face of both monkeys, using invasive electrophysiology (Cusick, Wall, \& Kaas, 1986; Dreyer et al., 1975; Manger, Woods, \& Jones, 1995) and humans, using microneurography during natural facial behaviors and/ or tactile stimulation (Johansson, Trulsson, Olsson, \& Abbs, 1988; Nordin \& Thomander, 1989; M. Trulsson \& Essick, 2010; Mats Trulsson \& Johansson, 2002). However, the inferred shape of facial receptive fields across the face is sparse and variable, and little is known with regard to the forehead. With regard to potential functional connections, several lines of evidence have shown functional linkages between sensorimotor representations of the face and hands (Gandevia \& Phegan, 1999; Gentilucci, Benuzzi, Gangitano, \& Grimaldi, 2001; Muret et al., 2014; Ramachandran, RogersRamachandran, Stewart, \& Pons, 1992; Serino, Padiglioni, Haggard, \& Làdavas, 2009). One recent study of tool useinduced plasticity, however, found no transfer of effect on tactile distance judgments from the hand to the face (Miller, Cawley-Bennett, Longo, \& Saygin, 2017a). Two other studies that measured plasticity induced by vibration-induced illusions of finger elongation (de Vignemont, Ehrsson, \& Haggard, 2005) and by arm immobilization (Bassolino, Finisguerra, Canzoneri, Serino, \& Pozzo, 2015) used the face as a comparison region for tactile distances on the hand. Because clear effects were found in both studies comparing the hand and face, any transfer between hand and face could not have been more than partial. Indeed, we are not aware of any studies that have reported transfer of effects on tactile distance judgments between the hand and the face. Moreover, while qualitatively similar anisotropies were reported on the forehead and hands by Longo et al. (2015), there was no correlation between these. Thus, the exact relation between anisotropies found on different body parts remains unclear.

The belly is the only body part that has been tested so far were no anisotropy has been found (Green, 1982; Longo et al., 2019; Marks et al., 1982). One could argue that tactile anisotropy is due to the use of a frame of reference within which the applied distances are estimated and compared, using for instance neighboring anatomical landmarks, which are absent in the belly. However, even if landmarks such as joints or facial features may affect tactile distance perception in general, it seems implausible that their use could produce the same type 
of anisotropies in body parts with very different types of landmarks, such as limbs and faces. Furthermore, there is empirical evidence that the magnitude of anisotropy is not necessarily correlated with the presence or absence of landmarks. For example, anisotropy is substantially smaller (or even absent) on the glabrous skin of the palm compared to the hairy skin of the hand dorsum (Longo, 2019; Longo et al., 2015; Longo \& Haggard, 2011), despite the number and location of landmarks being similar on both sides of the hand. Similarly, the magnitude of anisotropy is bigger on the forearm than on the hand dorsum (Le Cornu Knight et al., 2014), though there are more landmarks on the hand.

The distortions of tactile distance perception on the face may be related to other perceptual distortions. Studies of explicit body size estimation have generally reported overestimation of face width, using a range of measures including the moving-caliper procedure (Dolan, Birtchnell, \& Lacey, 1987; Halmi, Goldberg, \& Cunningham, 1977), the image-marking procedure (Meermann, 1983), and the adjustable light-beam apparatus (Dolce, Thompson, Register, \& Spana, 1987; Thompson \& Thompson, 1986). Overestimation of the width of the face has also been reported using a range of other tasks (Bianchi, Savardi, \& Bertamini, 2008; D'Amour \& Harris, 2017; Fuentes et al., 2013; Mora et al., 2018). It is an intriguing possibility that perceptual distortions such as the ones we have reported here may be linked to distortions of the conscious body image. Indeed, previous studies have provided evidence that tactile distance perception may be linked to higher-level body representations by showing that illusions of body size (de Vignemont et al., 2005; Tajadura-Jiménez et al., 2012; Taylor-Clarke et al., 2004) and tool use (Canzoneri et al., 2013; Miller et al., 2014; Miller, Longo, \& Saygin, 2017b) produce corresponding modulations in tactile distance perception.

In Experiment 1 there was a significant effect of laterality, with larger anisotropy on the left side of the face than on the right side. This effect was unpredicted, only marginally significant, and was not replicated in Experiment 2 (which did, however, find a non-significant trend in the same direction). Thus, we do not feel that any strong conclusion about laterality can be drawn. To our knowledge, only one previous study has compared tactile distance perception on the left and right sides of the body. Longo et al. (2015) found highly similar anisotropies on the left and right hands, with strong correlations between the two hands, and no hint of a laterality effect. The absence of a laterality effect for tactile distance on the hands mirrors the more general lack of differences between the two hands in tactile spatial acuity (Sathian \& Zangaladze, 1996; Vega-Bermudez \& Johnson, 2001).

Open Practices Statement The data for the experiments reported here are available at https://osf.io/y2gmf/ (Open Science Framework). None of the experiments were preregistered.
Acknowledgements This study was supported by European Research Council grant ERC-2013-StG-336050 under the FP7 granted to M.R.L. and by European Union Seventh Framework Programme (FP7-PEOPLE2011-IEF, 302277) granted to E.A.

\section{References}

Alloway, K. D., Rosenthal, P., \& Burton, H. (1989). Quantitative measurement of receptive field changes during antagonism of GABAergic transmission in primary somatosensory cortex of cats. Experimental Brain Research, 78, 541-532. https://doi.org/10. 1007/BF00230239

Anema, H. A., Wolswijk, V. W. J., Ruis, C., \& Dijkerman, H. C. (2008). Grasping Weber's illusion: The effect of receptor density differences on grasping and matching. Cognitive Neuropsychology, 25, 951967. https://doi.org/10.1080/02643290802041323

Bassolino, M., Finisguerra, A., Canzoneri, E., Serino, A., \& Pozzo, T. (2015). Dissociating effect of upper limb non-use and overuse on space and body representations. Neuropsychologia, 70, 385-392. https://doi.org/10.1016/j.neuropsychologia.2014.11.028

Bianchi, I., Savardi, U., \& Bertamini, M. (2008). Estimation and representation of head size (people overestimate the size of their head evidence starting from the 15th century). British Journal of Psychology, 99, 513-531. https://doi.org/10.1348/ 000712608X304469

Bizzozero, I., Costato, D., Della Sala, S., Papagno, C., Spinnler, H., \& Venneri, A. (2000). Upper and lower face apraxia: Role of the right hemisphere. Brain, 123, 2213-2230. https://doi.org/10.1093/brain/ 123.11.2213

Borenstein, M., Hedges, L. V., Higgins, J. P. T., \& Rothstein, H. R. (2009). Introduction to meta-analysis. Chichester, West Sussex: Wiley.

Boring, E. G. (1942). Sensation and perception in the history of experimental psychology. New York: Appleton-Century.

Brooks, V. B., Rudomin, P., \& Slayman, C. L. (1961). Peripheral receptive fields of neurons in the cat's cerebral cortex. Journal of Neurophysiology, 96, 27-39. https://doi.org/10.1152/jn.1961.24.3. 302

Calzolari, E., Azañón, E., Danvers, M., Vallar, G., \& Longo, M. R. (2017). Adaptation aftereffects reveal that tactile distance is a basic somatosensory feature. Proceedings of the National Academy of Sciences, 114, 4555-4560. https://doi.org/10.1073/pnas. 1614979114

Canzoneri, E., Ubaldi, S., Rastelli, V., Finisguerra, A., Bassolino, M., \& Serino, A. (2013). Tool-use reshapes the boundaries of body and peripersonal space representations. Experimental Brain Research, 228, 25-42. https://doi.org/10.1007/s00221-013-3532-2

Cholewiak, R. W. (1999). The perception of tactile distance: Influences of body site, space, and time. Perception, 28, 851-876. https://doi.org/ $10.1068 / \mathrm{p} 2873$

Cody, F. W. J., Garside, R. A. D., Lloyd, D., \& Poliakoff, E. (2008). Tactile spatial acuity varies with site and axis in the human upper limb. Neuroscience Letters, 433, 103-108. https://doi.org/10.1016/j. neulet.2007.12.054

Conti, F., Fabri, M., \& Manzoni, T. (1986). Bilateral receptive fields and callosal connectivity of the body midline representation in the first somatosensory area of primates. Somatosensory Research, 3, 273289. https://doi.org/10.3109/07367228609144588

Cusick, C. G., Wall, J. T., \& Kaas, J. H. (1986). Representations of the face, teeth and oral cavity in areas $3 \mathrm{~b}$ and I of somatosensory cortex in squirrel monkeys. Brain Research, 370, 359-364. https://doi.org/ 10.1016/0006-8993(86)90494-4 
D'Amour, S., \& Harris, L. R. (2017). Perceived face size in healthy adults. PLOS ONE, 12, e0177349. https://doi.org/10.1371/journal. pone. 0177349

Dasilva, A. F. M., Becerra, L., Makris, N., Strassman, A. M., Gonzalez, R. G., Geatrakis, N., \& Borsook, D. (2002). Somatotopic activation in the human trigeminal pain pathway. Journal of Neuroscience, 22, 8183-8192. https://doi.org/10.1523/JNEUROSCI.22-18-08183. 2002

de Vignemont, F., Ehrsson, H. H., \& Haggard, P. (2005). Bodily illusions modulate tactile perception. Current Biology, 15, 1286-1290. https://doi.org/10.1016/j.cub.2005.06.067

de Vignemont, F., Majid, A., Jola, C., \& Haggard, P. (2008). Segmenting the body into parts: Evidence from biases in tactile perception. Quarterly Journal of Experimental Psychology, 62, 500-512. https://doi.org/10.1080/17470210802000802

DiCarlo, J. J., \& Johnson, K. O. (2002). Receptive field structure in cortical area $3 \mathrm{~b}$ of the alert monkey. Behavioural Brain Research, 135, 167-178. https://doi.org/10.1016/S0166-4328(02)00162-6

DiCarlo, J. J., Johnson, K. O., \& Hsiao, S. S. (1998). Structure of receptive fields in area $3 \mathrm{~b}$ of primary somatosensory cortex in the alert monkey. Journal of Neuroscience, 18, 2626-2645. https://doi.org/ 10.1523/JNEUROSCI.18-07-02626.1998

Dolan, B. M., Birtchnell, S. A., \& Lacey, J. H. (1987). Body image distortion in non-eating disordered women and men. Journal of Psychosomatic Research, 31, 385-391. https://doi.org/10.1016/ 0022-3999(87)90009-2

Dolce, J. J., Thompson, J. K., Register, A., \& Spana, R. E. (1987). Generalization of body size distortion. International Journal of Eating Disorders, 6, 401-408. https://doi.org/10.1002/1098108X(198705)6:3<401::AID-EAT2260060310>3.0.CO;2-Z

Dreyer, D. A., Loe, P. R., Metz, C. B., \& Whitsel, B. L. (1975). Representation of head and face in postcentral gyrus of the macaque. Journal of Neurophysiology, 38, 714-733. https://doi.org/10.1152/ jn.1975.38.3.714

Engel, M. M., \& Keizer, A. (2017). Body representation disturbances in visual perception and affordance perception persist in eating disorder patients after completing treatment. Scientific Reports, 7, 16184. https://doi.org/10.1038/s41598-017-16362-w

Fabri, M., Polonara, G., Salvolini, U., \& Manzoni, T. (2005). Bilateral cortical representation of the trunk midline in human first somatic sensory area. Human Brain Mapping, 25, 287-296. https://doi.org/ 10.1002/hbm.20099

Faul, F., Erdfelder, E., Land, A.-G., \& Buchner, A. (2007). G*Power 3: A flexible statistical power analysis program for the social, behavioral, and biomedical sciences. Behavior Research Methods, 39, 175-191. https://doi.org/10.3758/BF03193146

Fiori, F., \& Longo, M. R. (2018). Tactile distance illusions reflect a coherent stretch of tactile space. Proceedings of the National Academy of Sciences, 115, 1238-1243. https://doi.org/10.1073/ pnas. 1715123115

Fuentes, C. T., Runa, C., Blanco, X. A., Orvalho, V., \& Haggard, P. (2013). Does my face FIT?: A face image task reveals structure and distortions of facial feature representation. PLOS ONE, 8, e76805. https://doi.org/10.1371/journal.pone.0076805

Gandevia, S. C., \& Phegan, C. M. L. (1999). Perceptual distortions of the human body image produced by local anaesthesia, pain and cutaneous stimulation. Journal of Physiology, 514, 609-616. https://doi. org/10.1111/j.1469-7793.1999.609ae.x

Gentilucci, M., Benuzzi, F., Gangitano, M., \& Grimaldi, S. (2001). Grasp with hand and mouth: A kinematic study on healthy subjects. Journal of Neurophysiology, 86, 1685-1699. https://doi.org/10. $1152 /$ jn.2001.86.4.1685

Goudge, M. E. (1918). A qualitative and quantitative study of Weber's illusion. American Journal of Psychology, 29, 81-119. https://doi. org/10.2307/1414107
Green, B. G. (1982). The perception of distance and location for dual tactile pressures. Perception and Psychophysics, 31, 315-323. https://doi.org/10.3758/BF03202654

Halmi, K. A., Goldberg, S. C., \& Cunningham, S. (1977). Perceptual distortion of body image in adolescent girls: Distortion of body image in adolescence. Psychological Medicine, 7, 253-257. https://doi.org/10.1017/S0033291700029330

Hidaka, S., Tucciarelli, R., Azañón, E., \& Longo, M. R. (2020). Tactile distance adaptation aftereffects do not transfer to perceptual hand maps. Acta Psychologica, 208, 103090. https://doi.org/10.1016/j. actpsy.2020.103090

Hubel, D. H., \& Wiesel, T. N. (1967). Cortical and callosal connections concerned with the vertical meridian of the visual field in the cat. Journal of Neurophysiology, 30, 1561-1573. https://doi.org/10. 1152/jn.1967.30.6.1561

Iannetti, G. ., Porro, C. ., Pantano, P., Romanelli, P., Galeotti, F., \& Cruccu, G. (2003). Representation of different trigeminal divisions within the primary and secondary human somatosensory cortex. NeuroImage, 19, 906-912. https://doi.org/10.1016/S10538119(03)00139-3

Iwamura, Y. (2000). Bilateral receptive field neurons and callosal connections in the somatosensory cortex. Philosophical Transactions of the Royal Society of London B, 355, 267-273. https://doi.org/10. 1098/rstb.2000.0563

Jain, N., Qi, H., Catania, K. C., \& Kaas, J. H. (2001). Anatomic correlates of the face and oral cavity representations in the somatosensory cortical area $3 \mathrm{~b}$ of monkeys. Journal of Comparative Neurology, 429, 455-468. https://doi.org/10.1002/1096-9861(20010115)429: $3<455::$ AID-CNE7>3.0.CO;2-F

Johansson, R. S., Trulsson, M., Olsson, K. A., \& Abbs, J. H. (1988). Mechanoreceptive afferent activity in the infraorbital nerve in man during speech and chewing movements. Experimental Brain Research, 72, 209-214. https://doi.org/10.1007/BF00248519

Jones, E. G., \& Powell, T. P. S. (1969a). Connexions of the somatic sensory pathway of the rhesus monkey. I. Ipsilateral cortical connexions. Brain, 92, 477-502. https://doi.org/10.1093/brain/92.3.477

Jones, E. G., \& Powell, T. P. S. (1969b). Connexions of the somatic sensory pathway of the rhesus monkey. II. Contralateral cortical connexions. Brain, 92, 717-730. https://doi.org/10.1093/brain/92. 4.717

Keizer, A., Aldegonda, M., Smeets, M., Christiaan, H., Hout, M. Van Den, Klugkist, I., ... Postma, A. (2011). Tactile body image disturbance in anorexia nervosa. Psychiatry Research, 190, 115-120. https://doi.org/10.1016/j.psychres.2011.04.031

Keizer, A., Smeets, M. A. M., Dijkerman, H. C., van Elburg, A., \& Postma, A. (2012). Aberrant somatosensory perception in anorexia nervosa. Psychiatry Research, 200, 530-537. https://doi.org/10. 1016/j.psychres.2012.05.001

Killackey, H. P., Gould, H. J., Cusick, C. G., Pons, T. P., \& Kaas, J. H. (1983). The relation of corpus callosum connections to architectonic fields and body surface maps in sensorimotor cortex of new and old world monkeys. Journal of Comparative Neurology, 219, 384-419. https://doi.org/10.1002/cne.902190403

Le Cornu Knight, F., Longo, M. R., \& Bremner, A. J. (2014). Categorical perception of tactile distance. Cognition, 131, 254-262. https://doi. org/10.1016/j.cognition.2014.01.005

Le Cornu Knight, F., Cowie, D., \& Bremner, A. J. (2017). Part-based representations of the body in early childhood: Evidence from perceived distortions of tactile space across limb boundaries. Developmental Science, 20, e12439. https://doi.org/10.1111/desc. 12439

Longo, M. R. (2017). Hand posture modulates perceived tactile distance. Scientific Reports, 7, 9665. https://doi.org/10.1038/s41598-01708797-y

Longo, M. R. (2019). Tactile distance anisotropy on the palm: A metaanalysis. Attention, Perception, \& Psychophysics. 
Longo, M. R., \& Golubova, O. (2017). Mapping the internal geometry of tactile space. Journal of Experimental Psychology: Human Perception and Performance, 43, 1815-1827. https://doi.org/10. 1037/xhp0000434

Longo, M. R., \& Haggard, P. (2011). Weber's illusion and body shape: Anisotropy of tactile size perception on the hand. Journal of Experimental Psychology: Human Perception and Performance, 37, 720-726. https://doi.org/10.1037/a0021921

Longo, M. R., \& Morcom, R. (2016). No correlation between distorted body representations underlying tactile distance perception and position sense. Frontiers in Human Neuroscience, 10, 593. https://doi. org/10.3389/fnhum.2016.00593

Longo, M. R., \& Sadibolova, R. (2013). Seeing the body distorts tactile size perception. Cognition, 126, 475-481. https://doi.org/10.1016/j. cognition.2012.11.013

Longo, M. R., Ghosh, A., \& Yahya, T. (2015). Bilateral symmetry of distortions of tactile size perception. Perception, 44, 1251-1262. https://doi.org/10.1177/0301006615594949

Longo, M. R., Lulciuc, A., \& Sotakova, L. (2019). No evidence of tactile distance anisotropy on the belly. Royal Society Open Science, 6 , 180866. https://doi.org/10.1098/rsos.180866

Mancini, F., Bauleo, A., Cole, J., Lui, F., Porro, C. A., Haggard, P., \& Iannetti, G. D. (2014). Whole-body mapping of spatial acuity for pain and touch. Annals of Neurology, 75, 917-924. https://doi.org/ 10.1002/ana.24179

Manger, P. R., Woods, T. M., \& Jones, E. G. (1995). Representation of the face and intraoral structures in Area $3 \mathrm{~b}$ of the squirrel monkey (Saimiri sciureus) somatosensory cortex, with special reference to the ipsilateral representation. Journal of Comparative Neurology, 363, 597-607. https://doi.org/10.1002/cne.903620412

Manzoni, T., Barbaresi, P., Conti, F., \& Fabri, M. (1989). The callosal connections of the primary somatosensory cortex and the neural bases of midline fusion. Experimental Brain Research, 76, 251266. https://doi.org/10.1007/BF00247886

Marks, L. E., Girvin, J. P., Quest, D. O., Antunes, J. L., Ning, P., O'Keefe, M. D., \& Dobelle, W. H. (1982). Electrocutaneous stimulation II. The estimation of distance between two points. Perception \& Psychophysics, 32, 529-536. https://doi.org/10.3758/ BF03204206

Meermann, R. (1983). Experimental investigation of disturbances in body image estimation in anorexia nervosa patients, and ballet and gymnastic pupils. International Journal of Eating Disorders, 2, 91100. https://doi.org/10.1002/1098-108X(198322)2:4<91::AIDEAT2260020416>3.0.CO;2-Z

Miller, L. E., Longo, M. R., \& Saygin, A. P. (2014). Tool morphology constrains the effects of tool use on body representations. Journal of Experimental Psychology: Human Perception and Performance, 40, 2143-2153. https://doi.org/10.1037/a0037777

Miller, L. E., Longo, M. R., \& Saygin, A. P. (2016). Mental body representations retain homuncular shape distortions: Evidence from Weber's illusion. Consciousness and Cognition, 40, 17-25. https:// doi.org/10.1016/j.concog.2015.12.008

Miller, L. E., Cawley-Bennett, A., Longo, M. R., \& Saygin, A. P. (2017a). The recalibration of tactile perception during tool-use is body-part specific. Experimental Brain Research, 235, 2917 2926. https://doi.org/10.1007/s00221-017-5028-y

Miller, L. E., Longo, M. R., \& Saygin, A. P. (2017b). Visual illusion of tool use recalibrates tactile perception. Cognition, 162, 32-40. https://doi.org/10.1016/j.cognition.2017.01.022

Mölbert, S. C., Sauer, H., Dammann, D., Zipfel, S., Teufel, M., Junne, F., ... Mack, I. (2016). Multimodal body representation of obese children and adolescents before and after weight-loss treatment in comparison to normal-weight children. PLOS ONE, 11, e0166826. https://doi.org/10.1371/journal.pone.0166826
Mora, L., Cowie, D., Banissy, M. J., \& Cocchini, G. (2018). My true face: Unmasking one's own face representation. Acta Psychologica, 191, 63-68. https://doi.org/10.1016/j.actpsy.2018.08.014

Moulton, E. A., Pendse, G., Morris, S., Aiello-Lammens, M., Becerra, L., \& Borsook, D. (2009). Segmentally arranged somatotopy within the face representation of human primary somatosensory cortex. Human Brain Mapping, 30, 757-765. https://doi.org/10.1002/hbm.20541

Mountcastle, V. B. (1957). Modality and topographic properties of single neurons of cat's somatic sensory cortex. Journal of Neurophysiology, 20, 408-434. https://doi.org/10.1152/jn.1957.20. 4.408

Mountcastle, V. B. (2005). The sensory hand: Neural mechanisms of somatic sensation. Cambridge, MA: Harvard University Press.

Muret, D., Dinse, H. R., Macchione, S., Urquizar, C., Farnè, A., \& Reilly, K. T. (2014). Touch improvement at the hand transfers to the face. Current Biology, 24, R736-R737. https://doi.org/10.1016/j.cub. 2014.07.021

Nordin, M., \& Thomander, L. (1989). Intrafascicular multi-unit recordings from the human infra-orbital nerve. Acta Physiologica Scandinavica, 135, 139-148. https://doi.org/10.1111/j.1748-1716. 1989.tb08561.x

Pandya, D. N., \& Vignolo, L. A. (1969). Interhemispheric projections of the parietal lobe in the rhesus monkey. Brain Research, 15, 49-65. https://doi.org/10.1016/0006-8993(69)90309-6

Penfield, W., \& Boldrey, E. (1937). Somatic motor and sensory representation in the cerebral cortex of man as studied by electrical stimulation. Brain, 60, 389-443. https://doi.org/10.1093/brain/60.4.389

Prins, N., \& Kingdom, F. A. A. (2009). Palamedes: Matlab routines for analyzing psychophysical data. http://www.palamedestoolbox.org.

Ramachandran, V. S., Rogers-Ramachandran, D., Stewart, M., \& Pons, T. P. (1992). Perceptual correlates of massive cortical reorganization. Science, 258, 1159-1160. https://doi.org/10.1126/science. 1439826

Sathian, K., \& Zangaladze, A. (1996). Tactile spatial acuity at the human fingertip and lip: Bilateral symmetry and inter-digit variability. Neurology, 46, 1995-1997. https://doi.org/10.1212/WNL.46.5. 1464

Sato, T., Okada, Y., Miyamoto, T., \& Fujiyama, R. (1999). Distributions of sensory spots in the hand and two-point discrimination thresholds in the hand, face and mouth in dental students, 93, 245-250.

Scarpina, F., Castelnuovo, G., \& Molinari, E. (2014). Tactile mental body parts representation in obesity. Psychiatry Research, 220(3), 960 969. https://doi.org/10.1016/j.psychres.2014.08.020

Serino, A., Padiglioni, S., Haggard, P., \& Làdavas, E. (2009). Seeing the hand boosts feeling on the cheek 5. Cortex, 45, 602-609. https://doi. org/10.1016/j.cortex.2008.03.008

Shanks, M. F., Pearson, R. C. A., \& Powell, T. P. S. (1985). The callosal connexions of the primary somatic sensory cortex in the monkey. Brain Research Reviews, 9, 43-65. https://doi.org/10.1016/01650173(85)90018-9

Spitoni, G. F., Serino, A., Cotugno, A., Mancini, F., Antonucci, G., \& Pizzamiglio, L. (2015). The two dimensions of the body representation in women suffering from anorexia nervosa. Psychiatry Research, 230, 181-188. https://doi.org/10.1016/j.psychres.2015. 08.036

Stone, K. D., Keizer, A., \& Dijkerman, H. C. (2018). The influence of vision, touch, and proprioception on body representation of the lower limbs. Acta Psychologica, 185, 22-32. https://doi.org/10.1016/j. actpsy.2018.01.007

Sur, M., Merzenich, M. M., \& Kaas, J. H. (1980). Magnification, receptive-field area, and size in areas $3 \mathrm{~b}$ and 1 of somatosensory cortex in owl monkeys. Journal of Neurophysiology, 44, 295-311. https://doi.org/10.1152/jn.1980.44.2.295

Tajadura-Jiménez, A., Väljamäe, A., Toshima, I., Kimura, T., Tsakiris, M., \& Kitagawa, N. (2012). Action sounds recalibrate perceived 
tactile distance. Current Biology, 22, R516-R517. https://doi.org/ 10.1016/j.cub.2012.04.028

Tamè, L., \& Longo, M. R. (2015). Inter-hemispheric integration of tactile-motor responses across body parts. Frontiers in Human Neuroscience, 9, 345. https://doi.org/10.3389/fnhum.2015.00345

Taylor-Clarke, M., Jacobsen, P., \& Haggard, P. (2004). Keeping the world a constant size: Object constancy in human touch. Nature Neuroscience, 7, 219-220. https://doi.org/10.1038/nn1199

Thompson, J. K., \& Thompson, C. M. (1986). Body size distortion and self-esteem in asymptomatic, normal weight males and females. International Journal of Eating Disorders, 5, 1061-1068. https:// doi.org/10.1002/1098-108X(198609)5:6<1061::AIDEAT2260050609>3.0.CO;2-C

Trulsson, M, \& Essick, G. K. (2010). Sensations evoked by microstimulation of single mechanoreceptive afferents innervating the human face and mouth. Journal of Neurophysiology, 103, 17411747. https://doi.org/10.1152/jn.01146.2009.

Trulsson, Mats, \& Johansson, R. S. (2002). Orofacial mechanoreceptors in humans: Encoding characteristics and responses during natural orofacial behaviors. Behavioural Brain Research, 135, 27-33. https://doi.org/10.1016/S0166-4328(02)00151-1

Ullrich, D. P., \& Woolsey, C. N. (1954). Trigeminal nerve representation in the "upper head area" of the postcentral gyrus of Macaca mulatta. Transactions of the American Neurological Association, 13, 23-28.

Vega-Bermudez, F., \& Johnson, K. O. (2001). Differences in spatial acuity between digits. Neurology, 56, 1389-1391. https://doi.org/ 10.1212/WNL.56.10.1389
Viechtbauer, W. (2010). Conducting meta-analyses in R with the metafor package. Journal of Statistical Software, 36, 1-48. https://doi.org/ 10.18637/jss.v036.i03

Vriens, J. P. M., \& van der Glas, H. W. (2009). Extension of normal values on sensory function for facial areas using clinical tests on touch and two-point discrimination. International Journal of Oral and Maxillofacial Surgery, 38, 1154-1158. https://doi.org/10.1016/ j.ijom.2009.06.006

Weber, E. H. (1834). De subtilitate tactus. In H. E. Ross \& D. J. Murray (Eds.), E. H. Weber on the tactile senses (pp. 21-128). London: Academic Press.

Won, S.-Y., Kim, H.-K., Kim, M.-E., \& Kim, K.-S. (2017). Two-point discrimination values vary depending on test site, sex and test modality in the orofacial region: A preliminary study. Journal of Applied Oral Science, 25, 427-435. https://doi.org/10.1590/16787757-2016-0462

Woolsey, C. N., Marshall, W. H., \& Bard, P. (1942). Representation of cutaneous tactile sensibility in the cerebral cortex of the monkey as indicated by evoked potentials. Bulletin of the Johns Hopkins Hospital, 70, 399-441.

Publisher's note Springer Nature remains neutral with regard to jurisdictional claims in published maps and institutional affiliations. 